\title{
Freedom isn't always free: immunoglobulin free light chains promote renal fibrosis
}

\author{
Erin B. Taylor ${ }^{1}$ and Michael J. Ryan ${ }^{1,2}$ \\ 'Department of Physiology and Biophysics, University of Mississippi Medical Center, Jackson, Mississippi, USA. 'G.V. (Sonny) Montgomery Veterans Affairs Medical Center, Jackson, Mississippi, USA.
}

\begin{abstract}
Multiple myeloma (MM) is a relatively common hematologic malignancy, and up to half of patients with MM present with renal dysfunction at the time of diagnosis. MM-associated renal injury has been linked to an excess level of monoclonal immunoglobulin free light chains (FLCs) in the circulation; however, it is not clear how these FLCs drive renal pathology. In this issue of the $J C l$, Ying et al. unravel a novel mechanism by which FLCs mediate renal injury in $\mathrm{MM}$ by inducing fibrotic and inflammatory pathways in the kidney. Specifically, FLC-mediated production of $\mathrm{H}_{2} \mathrm{O}_{2}$ was shown to activate JAK2/STAT1 signaling, increase production of IL-1 $\beta$ via induction of capsase- 1 , and promote activation of TCF- $\beta$ via $\alpha v \beta 6$ integrin. Moreover, the authors identified a tryptophan residue within a specific monoclonal FLC that was required for optimal $\mathrm{H}_{2} \mathrm{O}_{2}$ production and downstream signaling. A better understanding of the drivers of $\mathrm{MM}$-associated renal injury has potential for the identification of promising therapeutic targets.
\end{abstract}

proximal tubular cells are unable to catabolize all of the FLCs. Those FLCs that are not catabolized move to the distal tubule, where they interact with Tamm-Horsfall protein, leading to the development of cast nephropathy (7). The formation of casts is not present in every patient and depends on the nephrotoxicity of the specific FLC. A less common manifestation of $\mathrm{MM}$ is Fanconi syndrome, which occurs when FLCs undergo homotypic polymerization and form crystals within the endolysosomal system. In addition, the high rate of FLC endocytosis by the renal tubular cells in $\mathrm{MM}$ induces the production of inflammatory cytokines, including IL-6, IL-8, and TNF- $\alpha$, via pathways involving NF- $\kappa \mathrm{B}$ and MAPK activation $(8,9)$. The inflammatory and fibrotic pathways that are induced by the endocytosis of FLCs are the subject of current research.

\section{Renal injury in multiple myeloma}

Clonal plasma cell dyscrasias, including monoclonal gammopathy of undetermined significance (MGUS), solitary plasmacytoma, immunoglobulin-mediated amyloidosis, and multiple myeloma (MM), are relatively common disorders, with $2 \%$ to $3 \%$ of patients over the age of 50 affected by premalignant MGUS. MGUS can progress to MM, which accounts for $12 \%$ to $13 \%$ of all hematologic malignancies in the United States (1). The complications of MM include hypercalcemia, anemia, bone disease, such as lytic lesions and/or severe osteopenia, and renal insufficiency. $\mathrm{MM}$ is the most common malignancy associated with end-stage renal disease (2), and, depending on the diagnostic criteria (serum creatinine level), 20\% to 50\% of patients have renal failure at diagnosis (3). Because of the high prevalence of renal involvement in $\mathrm{MM}$ and its documented importance as a prognostic indicator (4), an understanding of the mechanisms that lead to the development of renal injury in MM are needed.

The principal factor that contributes to renal injury in $\mathrm{MM}$ is excess monoclonal immunoglobulin free light chains (FLCs), which can be toxic, resulting in damage of various renal structures, especially the tubules (3). Kappa (к) and lambda $(\lambda)$ FLCs are normally produced by the lymphoid system during immunoglobulin synthesis and are freely filtered by the glomerulus and catabolized in the proximal tubule. Within the proximal tubule, FLCs are endocytosed via a receptormediated mechanism that involves megalin and cubilin $(5,6)$ and then hydrolyzed, with the resulting amino acids returned to the circulation. Under normal conditions, the bulk of FLCs are catabolized, and the levels of FLCs present in urine are very low (1-10 mg/day). However, in the case of MM, the amount of circulating FLCs is markedly increased, and the

Related Article: p. 2792

Conflict of interest: The authors have declared that no conflict of interest exists.

\section{Linking FLCs to renal fibrosis}

The renal inflammatory pathways that are triggered by FLCs have been shown to be dependent on the ability of these proteins to catalyze the formation of hydrogen peroxide $\left(\mathrm{H}_{2} \mathrm{O}_{2}\right)$ from free water. This catalytic reaction was first shown for intact IgG molecules and Fab fragments by Wentworth et al. (10) and later for FLCs by Wang and Sanders (11). Similar to the differential effects of FLCs on the induction of cast nephropathy and Fanconi syndrome, distinct monoclonal FLCs differ in their abilities to catalyze $\mathrm{H}_{2} \mathrm{O}_{2}$ formation. In this issue, Ying et al. have identified a mechanism whereby monoclonal immunoglobulin FLCs activate inflammatory and fibrotic signaling pathways and show that this activation stems from the ability to generate $\mathrm{H}_{2} \mathrm{O}_{2}$ (12). Incubation of cultured human kidney epithelial cells with monoclonal preparations of $\kappa$ or $\lambda$ light chains promoted activation of JAK2 and STAT1. Further investigation revealed that monoclonal FLCs activate caspase-1 and increase production of IL-1 $\beta$ and active TGF- $\beta$. Activation of TGF- $\beta$ also involved 
the expression of $\alpha v \beta 6$ integrin on kidney epithelial cells, which has been shown in other disease states (13). Ying and colleagues confirmed the link between the JAK/STAT signaling and $\mathrm{H}_{2} \mathrm{O}_{2}$ production by FLCs, as the addition of the $\mathrm{H}_{2} \mathrm{O}_{2}$ scavenger 1,3-dimethyl-2-thiourea to the media prevented JAK/STAT activation.

For many of the renal complications associated with $\mathrm{MM}$, the structure of the FLC variable region $\left(\mathrm{V}_{\mathrm{L}}\right)$ determines its ability to induce renal pathology. For example, FLCs that cause Fanconi syndrome are generally of the $\kappa$ isotype and have nonpolar or hydrophobic residues in the complementarity-determining region (CDR) (14). Similarly, the CDR3 region determines binding to Tamm-Horsfall protein, thus demonstrating that certain monoclonal light chains are more likely to induce cast nephropathy in MM (15). Ying and colleagues generated a molecular model of a $\kappa$ FLC $V_{L}$ domain and discovered that a tryptophan (W) residue extends into the binding pocket. Using recombinant proteins, the authors elegantly showed that the recombinant $\mathrm{V}_{\mathrm{L}}$ with the $\mathrm{W}$ residue produced more $\mathrm{H}_{2} \mathrm{O}_{2}$ than did the $\mathrm{V}_{\mathrm{L}}$ with a W40G substitution. The recombinant $\mathrm{V}_{\mathrm{L}}$ with the native sequence also increased STAT1 activation and promoted IL-1 $\beta$ and TGF- $\beta$ production. The results of this study confirm the importance of amino acid sequence-specific effects of monoclonal FLCs on renal injury.

These in vitro findings were explored further in vivo. Specifically, Ying et al. injected Stat1 ${ }^{+/+}$and Stat1 $^{-/-}$mice with a monoclonal $\kappa 2$ light chain for 10 days. The $\kappa 2$ FLC localized to the proximal tubule brush border in both Stat1 ${ }^{+/+}$and Stat1/mice, but only induced albuminuria and increased urinary excretion of the tubular epithelial cell injury marker KIM-1 in $\mathrm{Stat1}^{+/+}$mice. The pathway leading to caspase-1 activation was also confirmed in vivo. While these studies were relatively short term and not extended to a time point at which the mice had elevated serum creatinine and declining renal function, they offer an important proof of concept supporting the cell culture studies. Importantly, these results support the idea that monoclonal FLCs in the proximal tubule promote a proinflammatory and profibrotic state that involves IL-1 $\beta$ and TGF- $\beta$ early in disease progression. Although not shown in this study, mounting evidence points to a central role for IL- $1 \beta$ as a potent inflammatory mediator in the kidney that triggers cell stress and the expression of inflammatory mediators (16). Recently, IL-1 $\beta$ was also shown to regulate the metabolism of kidney stromal cells, thereby leading to the initiation of fibrosis (17). TGF- $\beta$ has long been recognized as a fibrosis mediator; however, this cytokine can also induce protective and antiinflammatory effects (18). Taken together, the results of the study highlight a potentially important link between monoclonal FLCs and the initiation of renal inflammatory and fibrotic pathways in MM.

\section{Clinical implications}

The median survival for patients with MM is approximately three years (19), and renal impairment greatly reduces survival in this patient population. Clinical data suggest that a prompt reduction in circulating FLCs, either by the use of chemotherapeutic agents or direct removal from the plasma, can lead to renal recovery $(20,21)$. In particular, the proteasome inhibitor bortezomib is the most effective chemotherapeutic agent for treating myeloma with renal injury (22). Plasma cells synthesize and assemble large quantities of immunoglobulins, generate the bulk of circulating FLCs, and are highly sensitive to proteasome inhibition (23). In addition, bortezomib inhibits both NF- $\mathrm{KB}$ and MAPK pathways (24), which have been shown to act downstream of FLCs in the kidney. The effect of bortezomib in MM with renal involvement has been well documented (22), and it has also been effective in ameliorating renal injury in autoantibodymediated diseases such as systemic lupus erythematosus (SLE) (25-27).

It should be noted that numerous autoimmune and inflammatory diseases, including SLE, rheumatoid arthritis, multiple sclerosis, and diabetes, feature elevated circulating polyclonal FLCs (28). The pathological significance of these polyclonal FLCs has not yet been fully explored, but it is likely that they contribute to renal injury in some of these diseases. Moreover, patients with chronic kidney disease (CKD) (approximately $14 \%$ of the population in the US) have impaired renal clearance of FLCs as their glomerular filtration rate decreases. Studies suggest that these poly- clonal FLCs may also contribute to tubular injury in patients with CKD (29). Thus, the present work by Ying et al. is important, because it substantially expands upon previous knowledge related to the pathogenic role of monoclonal FLCs within the kidney that may ultimately provide insight for the development of novel therapeutics.

\section{Acknowledgments}

Work by the authors is funded by a Veterans Affairs Merit Award (BX002604-0 1A2, to MJR); a National Research Service Award from the National Heart, Lung, and Blood Institute (NHLBI), NIH (F32HL137393, to EBT); and the NIH (P01HL051971, P20GM104357, U54GM115428, and T32HL105324-05, to the Department of Physiology, University of Mississippi Medical Center).

Address correspondence to: Michael J. Ryan, Department of Physiology and Biophysics, University of Mississippi Medical Center, 2500 North State Street, Jackson, Mississippi 39216-4505. Email: mjryan@ umc.edu.

1. Palumbo A, Anderson K. Multiple myeloma. N Engl J Med. 2011;364(11):1046-1060.

2. Tsakiris DJ, et al. Incidence and outcome of patients starting renal replacement therapy for end-stage renal disease due to multiple myeloma or light-chain deposit disease: an ERAEDTA Registry study. Nephrol Dial Transplant. 2010;25(4):1200-1206.

3. Dimopoulos MA, Kastritis E, Rosinol L, Bladé J, Ludwig H. Pathogenesis and treatment of renal failure in multiple myeloma. Leukemia. 2008;22(8):1485-1493.

4. Knudsen LM, Hjorth M, Hippe E. Renal failure in multiple myeloma: reversibility and impact on the prognosis. Nordic Myeloma Study Group. Eur J Haematol. 2000;65(3):175-181.

5. Batuman V, et al. Myeloma light chains are ligands for cubilin (gp280). Am J Physiol. 1998;275(2):F246-F254.

6. Klassen RB, Allen PL, Batuman V, Crenshaw $\mathrm{K}$, Hammond TG. Light chains are a ligand for megalin. J Appl Physiol. 2005;98(1):257-263.

7. Huang ZQ, Sanders PW. Localization of a single binding site for immunoglobulin light chains on human Tamm-Horsfall glycoprotein. J Clin Invest. 1997;99(4):732-736.

8. Sengul S, Zwizinski C, Batuman V. Role of MAPK pathways in light chain-induced cytokine production in human proximal tubule cells. Am J Physiol Renal Physiol. 2003;284(6):F1245-F1254.

9. Sengul S, Zwizinski C, Simon EE, Kapasi A, Singhal PC, Batuman V. Endocytosis of light chains induces cytokines through activation of NFkappaB in human proximal tubule cells. Kidney 
Int. 2002;62(6):1977-1988.

10. Wentworth AD, Jones LH, Wentworth P, Janda $\mathrm{KD}$, Lerner RA. Antibodies have the intrinsic capacity to destroy antigens. Proc Natl Acad Sci US A. 2000;97(20):10930-10935.

11. Wang PX, Sanders PW. Immunoglobulin light chains generate hydrogen peroxide. J Am Soc Nephrol. 2007;18(4):1239-1245.

12. Ying WZ, Li X, Rangarajan S, Feng W, Curtis LM, Sanders PW. Immunoglobulin light chains generate proinflammatory and profibrotic kidney injury. J Clin Invest. 2019;129(7):2792-2806.

13. Ma LJ, et al. Transforming growth factor-betadependent and -independent pathways of induction of tubulointerstitial fibrosis in beta6(-/-) mice. Am J Pathol. 2003;163(4):1261-1273.

14. Messiaen T, et al. Adult Fanconi syndrome secondary to light chain gammopathy. Clinicopathologic heterogeneity and unusual features in 11 patients. Medicine (Baltimore). 2000;79(3):135-154.

15. Ying WZ, Sanders PW. Mapping the binding domain of immunoglobulin light chains for Tamm-Horsfall protein. Am J Pathol. 2001;158(5):1859-1866.
16. Anders HJ. Of inflammasomes and alarmins: IL-1 $\beta$ and IL-1 $\alpha$ in kidney disease. J Am Soc Nephrol. 2016;27(9):2564-2575.

17. Lemos DR, et al. Interleukin-1. J Am Soc Nephrol. 2018;29(6):1690-1705.

18. Sureshbabu A, Muhsin SA, Choi ME. TGF- $\beta$ signaling in the kidney: profibrotic and protective effects. Am J Physiol Renal Physiol. 2016;310(7):F596-F606.

19. Kyle RA, et al. Review of 1027 patients with newly diagnosed multiple myeloma. Mayo Clin Proc. 2003;78(1):21-33.

20. Hutchison CA, et al. Early reduction of serum-free light chains associates with renal recovery in myeloma kidney. J Am Soc Nephrol. 2011;22(6):1129-1136.

21. Bladé J, et al. Renal failure in multiple myeloma: presenting features and predictors of outcome in 94 patients from a single institution. Arch Intern Med.1998;158(17):1889-1893.

22. Heher EC, et al. Kidney disease associated with plasma cell dyscrasias. Blood. 2010;116(9):1397-1404.

23. Meister S, et al. Extensive immunoglobulin production sensitizes myeloma cells for proteasome inhibition. Cancer Res. 2007;67(4):1783-1792.

24. Mitsiades CS, et al. The role of the bone marrow microenvironment in the pathophysiology of myeloma and its significance in the development of more effective therapies. Hematol Oncol Clin North Am. 2007;21(6):1007-1034.

25 . Neubert K, et al. The proteasome inhibitor bortezomib depletes plasma cells and protects mice with lupus-like disease from nephritis. Nat Med. 2008;14(7):748-755.

26. Alexander $\mathrm{T}$, et al. The proteasome inhibitior bortezomib depletes plasma cells and ameliorates clinical manifestations of refractory systemic lupus erythematosus. Ann Rheum Dis. 2015;74(7):1474-1478.

27. Taylor EB, Barati MT, Powell DW, Turbeville HR, Ryan MJ. Plasma cell depletion attenuates hypertension in an experimental model of autoimmune disease. Hypertension. 2018;71(4):719-728.

28. Brebner JA, Stockley RA. Polyclonal free light chains: a biomarker of inflammatory disease or treatment target? F1000 Med Rep. 2013;5:4.

29. Parasuraman R, et al. Contribution of polyclonal free light chain deposition to tubular injury. Am J Nephrol. 2013;38(6):465-474. 